\title{
Arterial hypertension. Does the J curve exist? And then?
}

\author{
Gian Francesco Mureddu \\ Italian Association for Cardiovascular Prevention and Rehabilitation, Department of Internal Medicine, \\ San Giovanni-Addolorata Hospital, Rome, Italy
}

\begin{abstract}
The concept that 'the lower the blood pressure (BP) achieved the better the outcome' rests on the demonstration of a direct relationship between BP and incident outcomes, down to levels of $115 \mathrm{mmHg}$ of systolic BP (sBP) and $75 \mathrm{mmHg}$ of diastolic BP (dBP) carried out in 1 million individuals from 61 cohorts recruited between 1950 and 1990 and followed for about 14 years. The alternative to the 'lower the better' concept is the hypothesis of a J-shaped relationship, according to which the benefits of reducing $\mathrm{sBP}$ or $\mathrm{dBP}$ to low values may be dangerous leading to an increase in total mortality and/or in CV outcomes. Data from contemporary epidemiologic observations, (CALIBER study), showed that the relationships between rising BP and increased incidence of outcomes is maintained even over 85 years of age without the evidence of a J-shaped association with any of the outcomes at any age strata. In the English Longitudinal Study of Ageing study (ELSA), a tailored analysis of octogenarians showed that the increase in mortality rates associated with $\mathrm{BP}$ appears at $\mathrm{sBP}<110 \mathrm{mmHg}$ and $\geq 170 \mathrm{mmHg}$. In randomized controlled trials (SPRINT, HYVET and INVEST), the $\mathbf{J}$ curve seems to concern patients with an extensive atherosclerotic burden. An impaired autoregulation of coronary blood flow (CBF) leading to a fall in diastolic BP and resulting in a lowering in the perfusion pressure distal to the epicardial coronary artery stenosis, can eventually lead to myocardial ischemia. Diastolic dysfunction can concur in worsening CBF in diastole. These features are often seen in elderly patients with heart failure with preserved ejection fraction. The steeper position of the slope of the end-systolic elastance can lead to
\end{abstract}

Corresponding author: Gian Francesco Mureddu, Italian Association for Cardiovascular Prevention and Rehabilitation, Cardiac Rehabilitation and Secondary Cardiovascular Prevention Unit, Cardiology 2 Unit, Department of Internal Medicine, San Giovanni-Addolorata Hospital, Via dell'Amba Aradam 9, 00184 Rome, Italy. Tel. +39.06 .77055537 - Fax +30.06 .77056715$ E-mail: mureddu.gianfra@gmail.com

Key words: Arterial hypertension; elderly people; blood pressure targets; excess mortality.

Received for publication: 24 April 2018

Accepted for publication: 23 May 2018

(C) Copyright G.F. Mureddu, 2018

Tipografia PI-ME Editrice, Italy

Monaldi Archives for Chest Disease 2018; 88:953

doi: 10.4081/monaldi.2018.953

This article is distributed under the terms of the Creative Commons Attribution Noncommercial License (by-nc 4.0) which permits any noncommercial use, distribution, and reproduction in any medium, provided the original author(s) and source are credited. dramatic increases and decreases in BP for the same change in afterload or preload. This may explain why elderly hypertensives are more prone to suffer of hypertensive crisis and/or hypotension than the younger ones. "Pseudo-hypertension" caused by structural sclerotic changes in the brachial artery wall may cause overtreatment related falls in blood pressure. Thus, the $\mathrm{J}$ curve exists but only in patients with multiple comorbidities and/or extensive atherosclerotic burden.

\section{The relationship between pressure values and cardiovascular outcomes}

The concept that 'the lower the blood pressure (BP) achieved the better the outcome' rests on the demonstration of a direct relationship between BP and incident outcomes, down to levels of $115 \mathrm{mmHg}$ of systolic BP (sBP) and $75 \mathrm{mmHg}$ of diastolic BP (dBP). These data come from the Prospective Studies Collaboration meta-analysis that was carried out in 1 million individuals aged 40-89 years free from cardiovascular diseases (CVD) at baseline from 61 cohorts recruited between 1950 and 1990 and followed for about 14 years. That population-based large study reported a log-linear associations of both $\mathrm{SBP}$ and $\mathrm{dBP}$ with death from ischemic heart disease and stroke, with no apparent threshold [1].

The classical alternative to the 'lower the better' concept is the hypothesis of a J-shaped relationship, according to which the benefits of reducing $\mathrm{sBP}$ or $\mathrm{dBP}$ to low values may be dangerous leading even to an increase in total mortality and/or in CV outcomes. This hypothesis rests on two main considerations: the evidence from physiology that there is a low BP threshold for organ blood-flow autoregulation, and (2) the classical belief viewing BP as a compensatory mechanism for preserving organ function, that is it occurs an adequate pressure level to maintain perfusion pressure [2]. This controversy has always divided the clinicians and the researchers into two schools of thought but it is still unresolved. Hence the appropriate treatment target for systolic blood pressure (SBP) in older patients with hypertension remains uncertain. In this review we will look at the problem of J-curve existence in older people, and the population will probably be at increased risk of presenting this phenomenon, in the light of recent data and evidence available.

\section{What are the evidences in the elderly? Population-based studies}

After the HYVET study [3], we learned that in individuals over the age of 80 , with a first PAS $\geq 160 \mathrm{mmHg}$ response, it is helpful to reduce PAS between 150 and $140 \mathrm{mmHg}$, provided that the patient is in good physical and mental condition [4]. However in the growing population of very old patients (aged $\geq 80$ years) with hypertension, evidence on the relationship between blood pressure and the incidence of adverse cardiovascular outcomes are limited. Data obtained in contemporary ageing population may be useful to enlighten the nature of the relationship between BP and incident CVD. In the (CArdiovascular research using LInked Bespoke studies and Electronic health Records 
(CALIBER) study, the associations of blood pressure with 12 different presentations of cardiovascular disease were analyzed in a cohort of 1.25 million patients aged between 30 and 95 years and initially free from CVD, using electronic health records from 1997 to 2010. The outcomes which have been taking into account were: stable angina, unstable angina, myocardial infarction, unheralded coronary heart disease death, heart failure, cardiac arrest/sudden cardiac death, transient ischemic attack, ischemic stroke or stroke not otherwise specified, subarachnoid hemorrhage, intracerebral hemorrhage, peripheral arterial disease and abdominal aortic aneurysm [5].

About 25\% of the entire cohort was taking BP-lowering drugs. The relationships between rising pressure levels and increased incidence of outcomes showed a continuous growth even over 85 years of age. During the follow-up (5 years), people with systolic BP of 90-114 mmHg and diastolic blood pressure of $60-74 \mathrm{~mm} \mathrm{Hg}$, showed the lowest risk for CVD, with no evidence of a J-shaped curve at lower blood pressures. This result was seen in any age group. Particularly in people above 80 years of age with sBP between $115-129 \mathrm{mmHg}$, the risk of developing stable angina ( $\mathrm{HR}=1.21,95 \% \mathrm{CI}$ : 1.06-1.39), incident AMI (HR=1.05, 95\% CI: 0.95-1.17) or incident heart failure (HR=1.05, 95\% CI: 1.02-1.08), were consistently lower than in the same aged people with $\mathrm{sBP} \geq 180 \mathrm{mmHg}$ (HR=3.86, 95\% CI: 2.12-7.04) for stable angina; $\mathrm{HR}=2.22$, (95\% CI: $1.48-3.32)$ for AMI and $\mathrm{HR}=1.58,95 \%$ CI: $1.24-2.02$ for heart failure. These results confirm those of the Prospective Studies Collaboration in a contemporary population extensively treated with blood pressure-lowering agents and underline the lack of any J-shaped associations with any of the outcomes at any age strata.

In an observation from the English Longitudinal Study of Ageing (ELSA), carried out on a sample of 2692 octogenarians living in private households in England, the relationship between BP levels and cardiovascular disease as well as all-cause mortality were investigated. The outcome measures were also hypertension prevalence, awareness, treatment and control. Participants were separated into eight deciles of $\mathrm{sBP}$ values (<110 mmHg, 110-119 mmHg, 120-129 mmHg, 130-139 $\mathrm{mmHg}, 140-149 \mathrm{mmHg}, 150-159 \mathrm{mmHg}, 160-169 \mathrm{mmHg}$, and $>169$ $\mathrm{mmHg}$ ). Among treated participants $\geq 80$ years the association between SBP and CVD mortality seems to follow a J-shaped curve, with increased point estimates for both low and high extremes of sBP. The lowest event rate was observed in the sBP range was between 140 and $149 \mathrm{mmHg}(\mathrm{HR}=1.04 ; 95 \% \mathrm{CI}: 0.60-1.78)$. The association of sBP with all-cause mortality seemed to follow an inverse J-shaped curve with the critical nadir sBP range between 160 and $169 \mathrm{~mm} \mathrm{Hg}(\mathrm{HR}=0.78$, 0.51-1.21). However the results of Cox regression analyses for octogenarians showed that neither the association of sBP with CVD nor that of sBP with all-cause mortality were statistically significant and an apparent sharp increase in mortality rates associated with sBP seems to appear at $\mathrm{sBP}<110 \mathrm{mmHg}$ and $\geq 170 \mathrm{mmHg}$ [6]. Another major question that may be raised is that although a high proportion of people aged $\geq 80$ years were aware of being hypertensive and were on treatment compared with those aged $<80$ years, a low proportion of people achieved recommended BP targets $(<150 / 90 \mathrm{~mm} \mathrm{Hg})$. Hence the lack of benefit that may be expected with low BP levels might be related also to an inadequate BP control.

\section{What are the evidences in the elderly? Randomized controlled trials}

The Systolic Blood Pressure Intervention Trial (SPRINT) was designed to evaluate the effects of an intensive pressure control (PAS $<120 \mathrm{mmHg}$ ) compared to a conventional control (PAS $<140 \mathrm{mmHg}$ ) in subjects with high cardiovascular risk (clinically manifest or subclin- ical CVD, chronic kidney disease with eGFR $<60 \mathrm{ml} / \mathrm{min} / 1.73 \mathrm{~m}^{2}$, risk of CVD of $15 \%$ or more estimated with the Framingham risk score, 75 years or older, the primary outcome (a composite of myocardial infarction, acute coronary syndrome, stroke, heart failure, or death from cardiovascular causes) was reached for both groups of age, but even better in participants with age $\geq 75$ years ( $\mathrm{HR}=0.67 ; 95 \% \mathrm{CI}: 0.51-0.86)$ than in those $<75$ years (HR=0.80; 95\% CI:0.64-1.00) [7]. Following these results, a SPRINT sub-study was built up with the aim of evaluating the effects of intensive care strategy $(<120 \mathrm{mmHg}$ ) compared with usual care strategy $(<140 \mathrm{mmHg})$ of $\mathrm{sBP}$ targets in hypertensive patients of 75 years or older without diabetes. The whole study group was constituted by 2636 participants (mean age, 79.9 years; $37.9 \%$ women) of whom 1317 were allocated in the intensive care arm and 1319 in the usual care arm. The primary outcome was a composite of nonfatal myocardial infarction, acute coronary syndrome not resulting in a AMI, nonfatal stroke, nonfatal acute heart failure, and death from cardiovascular causes. The secondary outcome was all-cause mortality [8]. Patients were also stratified for gait speed (timed 4-m walk performed twice at the participant's usual pace from a standing start) and frailty status at randomization was quantified using a validated 37 -item index [9]. At a median follow-up of 3.14 years, there was a significantly lower rate of the primary composite outcome (102 events in the intensive treatment group vs 148 events in the standard treatment group; (HR=0.66; 95\% CI: 0.51-0.85) and all-cause mortality (73 deaths vs 107 deaths, respectively; HR=0.67; 95\% CI: 0.49-0.91). Kaplan-Meier Curves for the primary cardiovascular disease outcome in participants by baseline frailty within each frailty stratum, showed that the absolute event rates were lower for the intensive treatment group. Results were similar when participants were stratified by gait speed, with the HRs in favor of the intensive treatment group in each gait speed stratum. The overall rate of serious adverse events was not different between treatment groups [ $48.4 \%$ in the intensive care group $v s 48.3 \%$ in the standard care group; HR=0.99 (95\% CI: 0.89-1.11)]. However, the overall decrease in the incidence of fatal and nonfatal major CV events and all-cause death was paid in terms of higher rates of hypotension in the intensive care arm (2.4\%) as compared with the usual care arm (1.4\%), (HR=1.71; 95\% CI: 0.97-3.09); 3.0\% vs 2.4\%, for syncope (HR:1.23; 95\% CI: $0.76-2.00)$, respectively; $4.0 \%$ vs $2.7 \%$ for electrolyte abnormalities (HR=1.51; 95\% CI:0.99-2.33) and 5.5\% vs $4.0 \%$ for acute renal failure (HR=1.41; 95\% CI: 0.98-2.04) respectively. Finally, in the intensive care arm there was and increase rate of injurious falls (5.5\%) as compared with the usual care arm (4.9\%) (HR=0.91; 95\% CI: 0.65-1.29).

The observation that there is no evidence that frailty modifies the positive impact of antihypertensive treatment in very elderly people was confirmed in a sub-study of the HYpertension in the Very Elderly Trial (HYVET) which studied specifically the impact of frailty upon treatment effect in patients with hypertension aged 80 and over. Participants in HYVET were randomized 1:1 to active treatment with indapamide sustained release $1.5 \mathrm{mg} \pm$ perindopril 2 to $4 \mathrm{mg}$ or to matching placebo. Data relating to $\mathrm{BP}$, comorbidities, cognitive function, depression, and quality of life were collected at entry into the study and at subsequent follow-up visits. The frailty index (FI) was calculated at entry and showed a distribution similar to that seen in population studies of adults aged 80 years and above (median=0.17; IQR, $0.11-0.24$ ). Cox regression models did not find evidence of an interaction between effect of treatment for hypertension and frailty. Both the frailer and the fitter older adults with hypertension appeared to gain from treatment [10].

The $\mathrm{J}$ curve phenomenon seems to appear in sub-analysis of the INternational VErapamil SR Trandolapril Study (INVEST) [11] carried out to determine the influence of age on BP targets in patients with pre-existing coronary heart disease. The patients were divided into four age groups: 60 up to 60 years (6668), 60 to 69 years (7602), 70 to 79 years 
(6126), and >80 years (2180).The oldest patients tolerate a higher SBP better and a lower SBP worse compared with the younger patients. The J-curve between BP and all cause mortality and MI was observed with a $119 / 84 \mathrm{mmHg}$ nadir, but was not present for stroke (fatal and non-fatal) and among patients who were subjected to coronary revascularization. These observations, although often used to support the existence of the J-curve, actually reinforce the notion that the relationship between BP reduction and mortality concerns only some subgroups and basically its prognostic weight is inconsistent, except for patients with extensive atherosclerotic burden (like in those with an extensive coronary disease, not-revascularized or patients in whom coronary revascularization is not feasible). This may be possibly related to the compromised autoregulation of coronary blood flow (CBF) that the presence of a severe coronary disease may lead to a fall in diastolic BP that result in lowering the perfusion pressure distal to the epicardial coronary artery stenosis eventually leading to myocardial ischemia. This effect can be multiplied in the presence of multiple coronary stenoses. This mechanism may be even worse in the presence of hypertension and left ventricular hypertrophy [12].

Cardiovascular adaptations to arterial stiffening that occur with aging can also worsening this scenario. The increase in arterial stiffening which occurs in elderly persons, may determine earlier reflected pressure waves from the periphery to the proximal aorta; these waves produce an additional load (i.e. increased augmentation index) which in term, lead to an increased $\mathrm{LV}$ wall tension and afterload. This may concur together with the increase in pulse pressure, to a reduced CBF that may cause myocardial ischemia especially in thick walls of hypertrophied hearts. Diastolic dysfunction can develop in the context of concentric remodeling, increased wall thickness leading to decreased diastolic filling rate and also worsening coronary blood flow CBF in diastole [13].

This is what often happen in patients with heart failure with preserved ejection fraction (HFpEF) in which the position of the slope of the end-systolic pressure/volume relationship (end-systolic elastance; Ees) is steeper than in normal subjects leading to exaggerated increases and decreases in BP for the same change in afterload or preload. This may explain why elderly hypertensives are more inclined to suffer of hypertensive crisis and/or hypotension than normotensive or younger hypertensives [14].

Another cause of hypotension in the elderly have been explained in a recent scientific update of the European society of Hypertension focused on the treatment of high BP in elderly and octogenarians [15]. In the elderly a "pseudo-hypertension", caused by structural sclerotic changes in the brachial artery wall had been reported. Sluggish baroceptor function and reduced sensitivity to catecholamines may cause the elderly to be more sensitive to falls in BP either spontaneously or even induced by pharmacological therapy. Thus, can the effect of therapy explain the $\mathrm{J}$ curve phenomenon in the elderly? The relative risks for all causes of death in function of $\mathrm{dBP}$ in a treated population $\geq 85$-year-old are shown in a meta-analysis. Again, a J curve is observed, but when the results are adjusted for all comorbidities or health indicators, it disappeared. Thus, what makes it unsafe at dBP values $<75$ $\mathrm{mmHg}$ in the elderly does not seem to be related to self-medication or hypoperfusion of vital organs, but rather to the general clinical conditions. Potential risks of anti-hypertensive therapy however should always be taken well in mind. Physiologic changes that occur with aging may cause alterations on the absorption, distribution, metabolism and excretion of many anti-hypertensive drugs; so caution is mandatory when treating these patients in order to avoid accumulation and adverse effects due to overdosing [13]. Hence, as pointed put by the aforementioned ESH paper [15], in frail elderly patients it is recommended to base treatment decisions on comorbidity and carefully monitor the effects of treatment.
Finally does a J curve exist in the end stage of the life? A study presenting data for 144.403 people aged more than 80 years living in the UK from the Clinical Practice Research Datalink (CPRD), a large database of primary care, including about 7\% of UK General Practitioners tried to resolve the dispute if the antihypertensive treatment for octogenarians could be beneficial or harmful. The population sample was classified by frailty level and antihypertensive treatment status. Longitudinal analysis of patients' BP records showed that there is a 'terminal decline' in sBP in the 24 months before death, not accounted for by changes in antihypertensive treatment.

\section{Conclusions}

Blood pressure levels are related to prognosis in both elderly and oldest patients. Antihypertensive treatment is effective in improving prognosis even in the elderly. The effectiveness of therapy seems to be independent of frailty. Clinicians should be aware of: titrate the medications dosage gradually, recognize pseudo-hypertension, detect and correctly treat patients with comorbidities and/or extensive atherosclerotic burden. In conclusion, the $\mathrm{J}$ curve exists but it may be related more with comorbidities than with aging and/or frailty.

\section{References}

1. Lewington S, Clarke R, Qizilbash N, Peto R, Collins R, and the Prospective Studies Collaboration. Age-specific relevance of usual blood pressure to vascular mortality: a meta-analysis of individual data for one million adults in 61 prospective studies. Lancet 2002;360:1903-13.

2. Zanchetti A, Grassi G, Mancia G. When should antihypertensive drug treatment be initiated and to what levels should systolic blood pressure be lowered? A critical reappraisal. J Hypertens 2009;27:923-34.

3. Beckett NS, Peters R, Fletcher AE, et al. Treatment of hypertension in patients 80 years of age or older. N Engl J Med 2008;358:1887-98.

4. Mancia G, Fagard R, Narkiewicz K, et al. 2013 ESH/ESC Guidelines for the management of arterial hypertension: the Task Force for the Management of Arterial Hypertension of the European Society of Hypertension (ESH) and of the European Society of Cardiology (ESC). Eur Heart J 2013;34:2159-219.

5. Rapsomaniki E, Timmis A, George J, et al. Blood pressure and incidence of twelve cardiovascular diseases: lifetime risks, healthy life-years lost, and age-specific associations in 1.25 million people. Lancet 2014;383:1899-911.

6. Dregan A, Ravindrarajah R, Hazra N, et al. Longitudinal trends in hypertension management and mortality among octogenarians prospective cohort study. Hypertension 2016;68:97-105.

7. The SPRINT Research Group. A randomized trial of intensive versus standard blood-pressure control. N Engl J Med 2015;373:2103-16.

8. illiamson JD, Supiano MA, Applegate WB, et al. Intensive vs standard blood pressure control and cardiovascular disease outcomes in adults aged $\geq 75$ years. A randomized clinical trial. JAMA doi:10.1001/jama.2016.7050.

9. Pajewski NM, Williamson JD, Applegate WB, et al. Characterizing frailty status in the systolic blood pressure intervention trial. J Gerontol A Biol Sci Med Sci 2016;71:649-55.

10. Warwick J, Falaschetti E, Rockwood K, et al. No evidence that frailty modifies the positive impact of antihypertensive treatment in very elderly people: an investigation of the impact of frailty upon treatment effect in the HYpertension in the Very Elderly Trial 
(HYVET) study, a double-blind, placebo-controlled study of antihypertensives in people with hypertension aged 80 and over. BMC Med 2015;13:78.

11. Denardo SJ, Gong Y, Nichols WW, et al. Blood pressure and outcomes in very oldhypertensive coronary artery disease patients: an INVEST substudy. Am J Med 2010;123:719-26.

12. Angeli F, Reboldi G, Verdecchia P. Hypertension and the J-curve phenomenon: implications for tight blood pressure control. Hypertens Res 2013;36:109-11.

13. Aronow WS, Fleg JL, Pepine CJ, et al. ACCF/AHA 2011 expert consensus document on hypertension in the elderly: a report of the American College of Cardiology Foundation Task Force on Clinical Expert Consen- sus documents developed in collaboration with the American Academy of Neurology, American Geriatrics Society, American Society for Preventive Cardiology, American Society of Hypertension, American Society of Nephrology, Association of Black Cardiologists and European Society of Hypertension. J Am Coll Cardiol 2011;57:2037-114.

14. Borlaug BA, Paulus WJ. Heart failure with preserved ejection fraction: pathophysiology, diagnosis, and treatment. Eur Heart J 2011;32:670-9.

15. Kjeldsen SE, Stenehjem A, Os I, et al. Treatment of high blood pressure in elderly and octogenarians: European Society of Hypertension statement on blood pressure targets. Blood Pres DOI:10.1080/08037051.2016.1236329. 